\title{
Influence de l'alimentation programmée sur le comportement de prise d'eau chez le Chien
}

\author{
J.L. ARDISSON, C. DOLISI, Camille OZON et Dominique CRENESSE \\ Avec la collaboration technique de C. Birot et R. Governatori \\ Laboratoire de Physiologie \\ Faculté de Médecine de Nice \\ Chemin de Vallombrose, 06034 Nice Cedex (France)
}

\begin{abstract}
Résumé
Le comportement de prise d'eau de trois chiens a été étudié dans deux situations expérimentales :

1) Lorsqu'ils disposent d'aliments à volonté (alimentation ad libitum).

2) Lorsqu'ils disposent, une seule fois par jour, d'un poids fixe d'aliments, représentant 30,60 ou 90 p. 100 de leur prise quotidienne spontanée (alimentation programmée). La comparaison des résultats montre que la prise d'un repas par jour de poids déterminé :

1. Laisse persister :

a) les différences interindividuelles de la dimension des prises d'eau;

b) une liaison significative entre le poids des prises d'eau et les intervalles qui leur font suite chez deux chiens (tableau 4).

2. Modifie les patterns spontanés des prises d'eau selon un profil unique : celles-ci sont regroupées dans les heures qui suivent la prise d'aliments (figure 3 ).

3. Suscite :

a) une augmentation des débits des prises d'aliments (tableau 3) et une diminution de ceux des prises d'eau, pour les trois chiens (tableau 2);

b) une augmentation de la quantité d'eau ingérée par gramme d'aliments chez deux chiens (tableau 1).
\end{abstract}

\section{Introduction}

Les travaux se rapportant à l'étude des relations entre les comportements de prise d'eau et d'aliments des petits mammifères de laboratoire sont très nombreux et diversifiés. Généralement, la dépendance réciproque entre ces consommations a été montrée en plaçant les animaux dans une situation de contrainte : soit privation d'eau (Bing \& Mendel, 1931; AdolPh, 1947; Strominger, 1947; Verplanck \& Haye, 1953 ; Bolles, 1961 ; Crzek \& Nocenti, 1965 ; Kutscher, 1969 ; Prud’hoN et al., 1975), soit privation d'aliments (STrominger, 1947 ; Cizek \& Nocenti, 1965 ; Fitzsimons \& Le Magnen, 1969; OAtley, 1971). Les résultats présentés par ces auteurs sont généralement établis à partir de l'observation d'un grand nombre d'individus et expriment un comportement moyen de l'espèce. 
Plus récemment, certains auteurs ont réalisé des études individuelles du comportement alimentaire du Rat (Levitsky, 1974 ; BERNSTEIn, 1975) et du Singe (NATElson et Bonbrigit, 1978). Ces travaux ont montré l'eixstence de fortes différences interindividuelles même lorsque l'expérimentation utilisait des souches très homogènes.

Le présent travail se propose :

1) d'étudier le comportement d'ingestion d'eau de trois chiens en alimentation ad libitum et en alimentation programmée;

2) de comparer les résultats individuels obtenus au cours de chaque alimentation afin de vérifier si, chez cette espèce se retrouvent également de fortes différences d'un sujet à l'autre ;

3) d'étudier les relations fondamentales pouvant caractériser le comportement alimentaire du Chien en situation ad libitum et les modifications induites par l'alimentation programmée.

\section{Matériel et méthodes}

\section{1. - Pièce expérimentale}

La pièce dans laquelle vit l'animal a une surface de $30 \mathrm{~m}^{2}$. Elle est éclairée, en lumière artificielle, de $6 \mathrm{~h}$ du matin à $22 \mathrm{~h}$. La température ambiante est maintenue stable aux environs de $22{ }^{\circ} \mathrm{C}\left(20\right.$ à $\left.24{ }^{\circ} \mathrm{C}\right)$, l'humidité relative entre 50 et 70 p. cent. Le chien dispose d'une niche traditionnelle.

Deux compartiments indépendants, situés en dehors de la pièce, communiquent avec elle, chacun par un orifice de $40 \mathrm{~cm}$ de large et de $100 \mathrm{~cm}$ de hauteur barré, en permanence, par 4 faisceaux de lumière infra-rouge.

Chaque compartiment est occupé par une balance électronique à sortie BCD (Mettler PL 3000). Les récipients placés sur leur plateau contiennent l'un de l'eau ordinaire, l'autre des granulés secs (U.A.R. 121) contenant un maximum de 10 p. cent d'humidité.

Chaque fois que l'animal vient boire ou manger il interrompt les faisceaux de lumière infra-rouge ce qui déclenche instantanément l'impression des paramètres suivants (imprimante thermique Hewlett-Packard $5150 \mathrm{~A}$ ) : le temps en heure, minutes et secondes; la nature de la prise et le poids porté sur la balance. Lorsque le chien quitte le récipient d'eau ou d'aliment, le rétablissement des faisceaux lumineux entraîne à nouveau instantanément l'enregistrement des mêmes paramètres. Ainsi le poids et la durée de chaque prise alimentaire seront obtenus par simple différence des valeurs lues (ARDisson et al., 1979).

\section{2. - Les animaux}

Trois chiens adultes mâles ont été utilisés :

Le chien $\mathrm{n}^{\circ} 1(25 \mathrm{~kg})$ et le chien $\mathrm{n}^{\circ} 2(23 \mathrm{~kg})$ sont des bâtards d'origine rurale ; le chien $n^{\circ} 3(14 \mathrm{~kg})$ est un animal de race Beagle, acheté dans un centre d'élevage. 


\section{3. - Conditions de vie}

a) Lorsque les animaux arrivent au laboratoire, ils subissent un examen vétérinaire. Ils sont sélectionnés à l'issue d'une période d'observation de deux mois minimum durant laquelle ils sont familiarisés aux conditions des expériences (dispositions de la pièce de séjour, éclairage, contacts avec le personnel). Ce n'est qu'après avoir constaté simultanément la régularité des volumes des ingestions solides et hydriques journalières et la stabilité du poids corporel que les animaux sont définitivement retenus. La stabilité de ces mêmes paramètres est revérifiée systématiquement au cours d'une nouvelle période d'observation de contrôle, avant chaque expérimentation.

b) En dehors des périodes d'expérimentation, ils vivent dans des boxes de $10 \mathrm{~m}^{2}$ de surface, où ils disposent de la même nourriture que pendant les expériences.

\section{4. - Protocoles expérimentaux}

Les trois chiens ont subi chacun deux protocoles expérimentaux.

\section{a. - Alimentation ad libitum}

L'animal dispose d'eau et d'aliments à volonté. La durée totale de l'observation est de l'ordre de 45 jours. Les durées d'enregistrement continu varient de 2 à 5 jours. Elles sont séparées par des journées de sortie tirées au sort au cours desquelles le chien se trouve en liberté de $10 \mathrm{~h}$ à $14 \mathrm{~h}$ sur la terrasse du chenil, sans eau ni aliment.

\section{b. - Alimentation programmée}

L'animal disposant d'eau à volonté est soumis à trois programmes alimentaires. Au cours de ces programmes un poids d'aliments, représentant une fraction de la prise journalière spontanée moyenne calculée à l'occasion de la première série expérimentale, est proposé à chaque chien en une seule fois de $9 \mathrm{~h}$ à $10 \mathrm{~h}$. Cette fraction est égale à 30 p. 100 pour le programme « $\mathrm{A} », 60$ p. 100 pour le programme «B» et 90 p. 100 pour le programme «C». L'unité d'expérimentation dure 36 jours. Elle est constituée par la succession de 12 jours en programme «B», 12 jours en programme « $A$ » et 12 jours en programme « $C$ » sans aucune interruption. Trois unités d'expérimentation ont été imposées aux trois chiens. Comme pour le premier protocole les durées d'enregistrement continu sont séparées par des jours de sortie tirés au sort. Pendant la durée de l'unité d'expérimentation le chien reçoit, tous les jours sans exception, le poids d'aliments correspondant au programme auquel il est soumis.

L'évolution du poids corporel de chacun des trois chiens au cours de ce protocole expérimental est donnée par la figure 1. Les trois programmes $\mathrm{A}$ de chaque chien ont été comparés. Aucune différence significative du comportement de prise d'eau n'a pu être décelée à partir des données recueillies.

Pour les deux protocoles les jours de sortie et ceux émaillés d'un incident technique ne sont pas pris en compte lors de l'exploitation des données, ce qui explique le nombre variable de «jours utiles $»$ retenus pour chaque chien et chaque programme. 

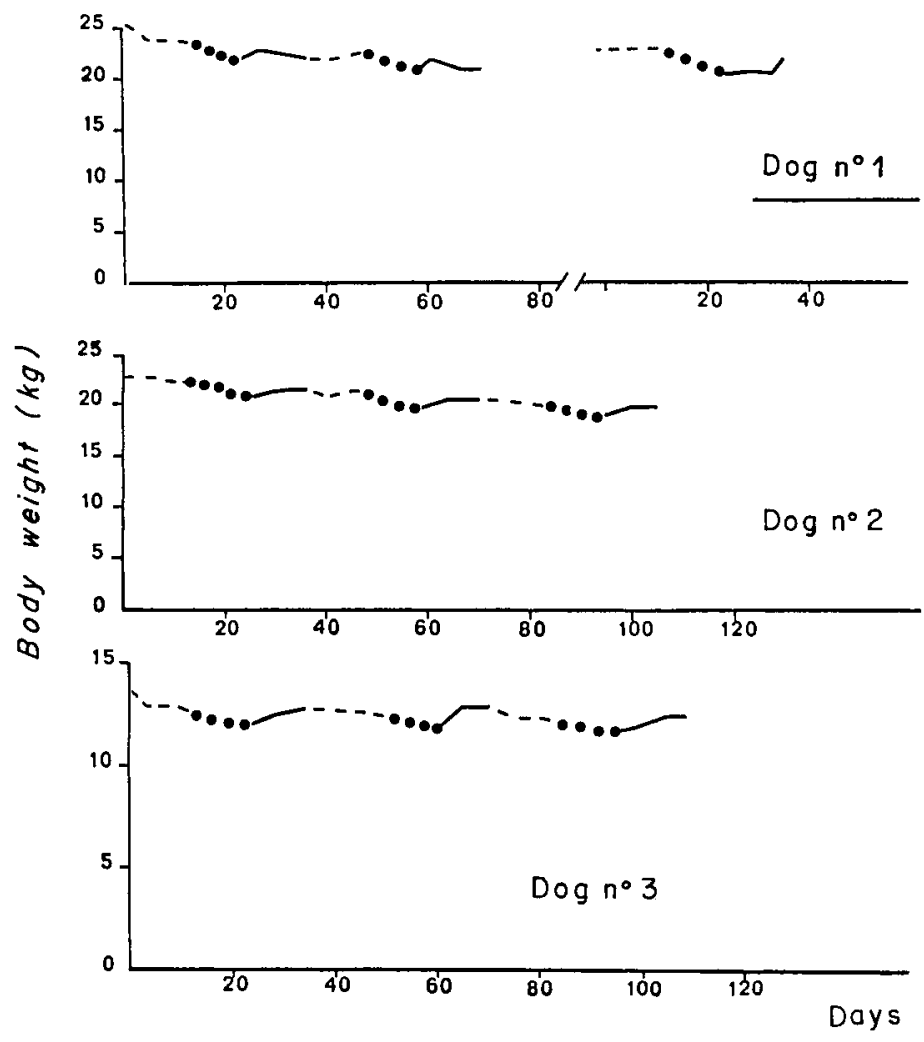

FIG. 1

Evolution $d u$ poids corporel des trois chiens en alimentation programmée

Body weight changes in the three dogs during programmed feeding

Valeurs du poids corporel mesuré lors de chaque jour de sortie.

The dogs were weighed each time they were taken out of experimental setting.

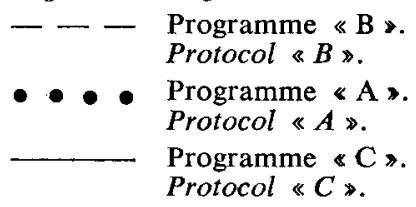

\section{Résultats}

\section{1. - Résultats journaliers}

En situation ad libitum le poids d'eau consommée, par gramme d'aliments ingéré, varie de 2,10 g à 2,49 g selon l'animal considéré (tableau 1). Les consommations journalières spontanées d'aliments et d'eau du chien $n^{\circ} 3$ sont significativement plus élevées que celles des deux autres chiens ( $\mathrm{p}<1$ p. 1000 ). 


\section{Tableau 1}

Poids d'aliments et d'eau ingérés par jour et valeurs du rapport de ces deux poids Weight of food (F) and water ingested (W) per day and $\frac{W}{F}$ ratios

\begin{tabular}{|c|c|c|c|c|}
\hline 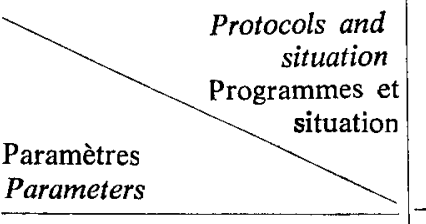 & A & B & $\mathrm{C}$ & Ad libitum \\
\hline \multirow{2}{*}{$\begin{array}{l}\text { Dog } n^{\circ} 1 \\
\text { Chien } n^{\circ} 1 \\
\text { Quantité de granulés ingérée } \\
\text { par jour } / \mathrm{kg}(\mathrm{G}) \text { en gram- } \\
\text { mes (1) }\end{array}$} & & & & \\
\hline & $\begin{array}{c}4,8 \\
n=26\end{array}$ & $\begin{array}{c}9,6 \\
n=36\end{array}$ & $\begin{array}{c}14,4 \\
n=29\end{array}$ & $\begin{array}{c}14,91 \pm 1,01 \\
n=25\end{array}$ \\
\hline $\begin{array}{l}\text { Quantité d'eau ingérée par } \\
\text { jour } / \mathrm{kg} \text { (E) en grammes } \\
(2)\end{array}$ & $\begin{array}{c}22,77 \pm 1,51 \\
n=26 \\
* * *\end{array}$ & $\begin{array}{c}27,80 \pm 0,93 \\
n=36 \\
* *\end{array}$ & $\begin{array}{c}47,54 \pm 1,54 \\
n=29 \\
* *\end{array}$ & $\begin{array}{l}37,13 \pm 3,07 \\
n=25\end{array}$ \\
\hline$\frac{\mathrm{E}}{\mathrm{G}} \ldots \ldots \ldots \ldots \ldots \cdots \cdots$ & 4,74 & 2,89 & 3,30 & 2,49 \\
\hline \multirow{2}{*}{$\begin{array}{l}\text { Dog } n^{\circ} 2 \\
\text { Chien } \mathrm{n}^{\circ} 2 \\
\text { Quantité de granulés ingérée } \\
\text { par jour } / \mathrm{kg}(\mathrm{G}) \text { en gram- } \\
\text { mes }\end{array}$} & & & & \\
\hline & $\begin{array}{c}5,22 \\
n=24\end{array}$ & $\begin{array}{l}10,44 \\
n=26\end{array}$ & $\begin{array}{c}15,66 \\
n=18\end{array}$ & $\begin{array}{c}16,98 \pm 0,94 \\
n=25\end{array}$ \\
\hline $\begin{array}{l}\text { Quantité d'eau ingérée par } \\
\text { jour } / \mathrm{kg} \text { (E) en grammes }\end{array}$ & $\begin{array}{c}26,52 \pm 1,38 \\
n=24 \\
* * *\end{array}$ & $\begin{array}{c}34,20 \pm 1,51 \\
n=26\end{array}$ & $\begin{array}{c}55,66 \pm 2,13 \\
n=18 \\
* * *\end{array}$ & $\begin{array}{l}37,47 \pm 2,26 \\
n=25\end{array}$ \\
\hline$\frac{\mathbf{E}}{\mathrm{G}} \ldots \ldots \ldots \ldots \ldots \cdots \cdots$ & 5,08 & 3,28 & 3,55 & 2,20 \\
\hline \multicolumn{5}{|l|}{$\begin{array}{l}\text { Dog } n^{\circ} 3 \\
\text { Chien } n^{\circ} 3\end{array}$} \\
\hline $\begin{array}{l}\text { Quantité de granulés ingérée } \\
\text { par jour } / \mathrm{kg}(G) \text { en gram- } \\
\text { mes }\end{array}$ & $\begin{array}{c}7,14 \\
n=29\end{array}$ & $\begin{array}{c}14,28 \\
n=31\end{array}$ & $\begin{array}{c}21,42 \\
n=28\end{array}$ & $\begin{array}{c}23,54 \pm 1,15 \\
n=25\end{array}$ \\
\hline $\begin{array}{l}\text { Quantité d'eau ingérée par } \\
\text { jour } / \mathrm{kg} \text { (E) en grammes }\end{array}$ & $\begin{array}{c}13,43 \pm 0,85 \\
n=29 \\
* * *\end{array}$ & $\begin{array}{c}24,90 \pm 1,10 \\
n=31 \\
* * *\end{array}$ & $\begin{array}{c}37,39 \pm 1,88 \\
n=28 \\
* * *\end{array}$ & $\begin{array}{l}49,41 \pm 3,16 \\
n=25\end{array}$ \\
\hline$\frac{E}{G}$ & 1,88 & 1,74 & 1,745 & 2,10 \\
\hline $\begin{array}{rl}* * & \mathrm{p}<0,01 \\
* * * & \mathrm{p}<0,001\end{array}$ & & & & \\
\hline $\begin{array}{l}\text { (1) Daily pellet intake } / \mathrm{kg} \\
\text { (2) Daily water intake } / \mathrm{kg}\end{array}$ & $\begin{array}{l}\text { in gramms. } \\
\text { in gramms. }\end{array}$ & & & \\
\hline
\end{tabular}




\section{TABLEAU 1 (suite)}

Les valeurs de la situation ad libitum sont les moyennes de 25 jours d'observation ( \pm erreur standard à la moyenne).

$\mathrm{Au}$ cours des programmes alimentaires « $\mathrm{A}$ », «B» et « $\mathrm{C} »$ chaque chien fait un seul repas par jour. Ce repas débute toujours à 9 heures. Il est constitué par un poids fixe d'aliments représentant une fraction de sa prise journalière moyenne en situation ad libitum. Cette fraction est égale à 30 p. 100 pour le programme «A», 60 p. 100 pour le programme «B» et 90 p. 100 pour le programme $« \mathrm{C} »$. Les poids d'aliments ingérés par jour sont donc constants pour un même programme.

$\mathrm{Au}$ cours de ces programmes l'eau est disponible à volonté et les valeurs indiquées sont des valeurs moyennes ( \pm erreur standard à la moyenne).

Les * du tableau indiquent les résultats des comparaisons entre les quantités d'eau ingérées au cours des programmes « $\mathbf{A} »$, « $\mathbf{B}$ » et « $\mathbf{C}$ » et la situation ad libitum.

The ad libitum values are averages of 25 observation days ( \pm S.E.M.).

During the feeding protocols « $A », 《 B »$ and «C», each dog ingested one meal per day always beginning at 9.00 a.m. This meal was composed of a fixed weight of food representing a fraction of the dog's daily voluntary mean intake. This fraction equals $30 \mathrm{p}$. 100 in protocol $A \gg$, $60 \mathrm{p} .100$ in protocol $« B »$ and $90 \mathrm{p} .100$ in protocol $" C »$. The weight of food ingested per day is constant within each protocol.

Water was freely available and the values indicated are averages \pm S.E.M.

The : in the table indicate comparison of the quantity of water ingested daily in protocols « $A », \ll B »$ and $《 C »$ and in the ad libitum situation.

Chez les trois chiens la réduction de la quantité d'aliments disponible par jour entraîne en programmes « $A$ » et « $B$ » une diminution de la consommation d'eau. Chez les chiens $n^{\prime \prime s} 1$ et 2 cette consommation augmente en programme $C$ (figure 2). Cependant, l'évolution du rapport de ces deux paramètres montre bien que la réduction hydrique spontanée n`est pas proportionnelle à la réduction alimentaire imposée (tableau 1). En effet, au cours des trois programmes alimentaires, ce rapport est toujours supérieur à celui de la situation ad libitum. Cette relative surconsommation d'eau est maximale pour le programme « $A »$, le plus restrictif.

Ces phénomènes ne sont pas retrouvés chez le chien $\mathrm{n}^{n} 3$ qui présente une prise moyenne journalière croissant régulièrement $\mathrm{du}$ programme « $\mathrm{A}$ » à la situation $a d$ libitum (tableau 1).

La comparaison interindividuelle de la quantité d'eau ingérée par jour et par kilogramme de poids corporel montre que pour le programme alimentaire " $A$ » le chien $n^{\circ} 3$ diffère très significativement des deux autres ( $p$ toujours inférieur à 1 p. 1000 ). En programmes alimentaires « $B$ » et « $C$ » les trois chiens diffèrent tous très significativement les uns des autres ( $p$ compris entre 1 p. 1000 et 5 p. 100).

\section{2. - Evolution horaire de la fréquence moyenne des prises d'eau}

En situation ad libitum les profils horaires de la fréquence journalière des prises d'eau (fig. 3) montrent que les 3 chiens répartissent spontanément celles-ci sur l'ensemble du nycthémère. On peut remarquer, pour le chien $\mathrm{n}^{\circ} 2$, un nombre plus élevé de prises en début de phase d'éclairement et durant la phase d'obscurité. 

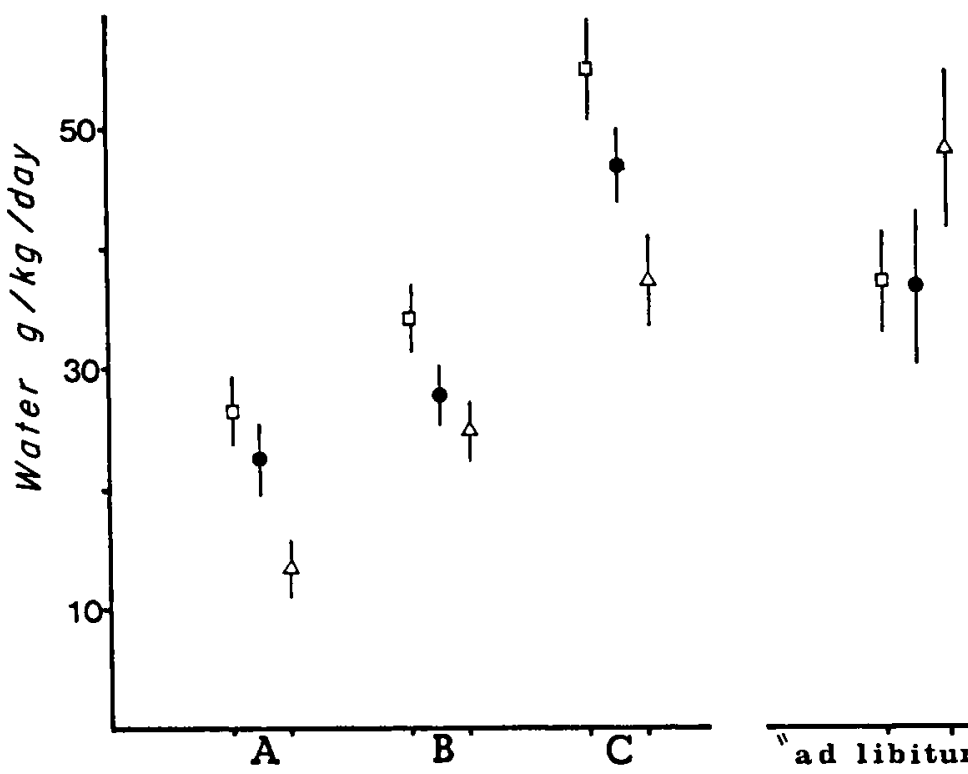

FIG. 2

Poids moyen d'eau ingérée par jour et par kilo de poids corporel

Average quantity of water ingested per day and per $\mathrm{kg}$ body weight

Valeurs calculées pour chaque chien au cours des trois programmes alimentaires et de la situation ad libitum.

Les barres verticales délimitent l'intervalle de confiance à 95 p. 100.

Values calculated for each dog during the three feeding protocols and in an ad libitum situation.

95 p. 100 confidence limit intervals are indicated.

- Chien $\mathrm{n}^{\circ} 1$.

Dog No. 1.

$\square \quad$ Chien $\mathrm{n}^{\circ} 2$.

Dog No. 2 .

$\triangle$ Chien $\mathrm{n}^{\circ} 3$.

Dog No. 3 .

L'imposition d'un repas unique modifie considérablement ces profils. Dans tous les cas, la courbe des fréquences présente un pic situé dans la tranche horaire du repas ou dans la suivante puis, une décroissance progressive dans les heures qui suivent. Lorsque le poids du repas diminue le nombre d'heures au cours desquelles on observe des prises d'eau se réduit : il passe de 9 heures en programme * $\mathrm{C}$ 》 à 5 heures en programme « A $\gg$. Dans tous les cas on n'observe que peu ou pas de prises d'eau en fin de phase d'éclairement, durant la phase d'obscurité et dans les heures qui précèdent l'heure de la prise alimentaire. 

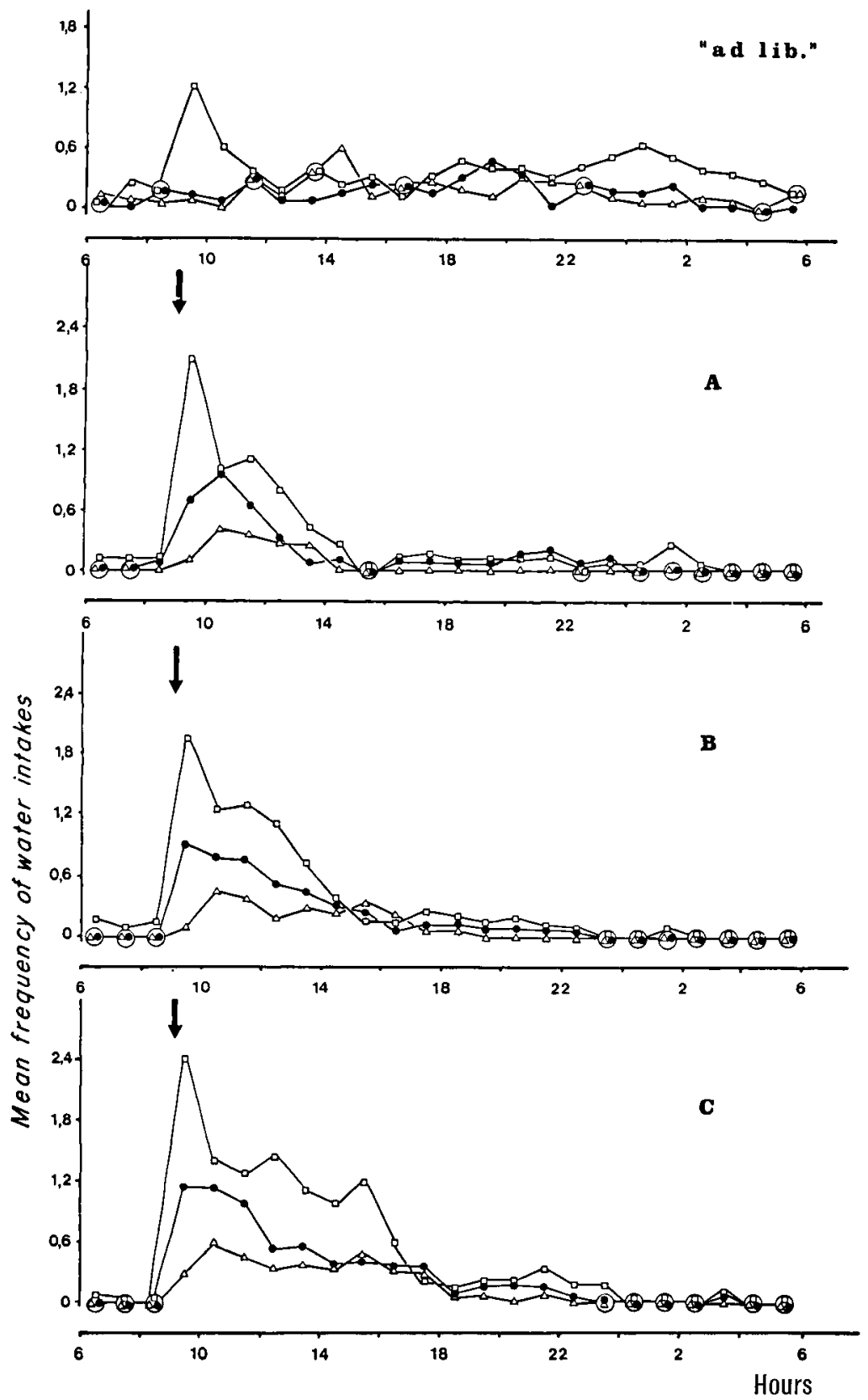

FIG. 3

Evolution horaire de la fréquence moyenne des prises d'eau Hourly distribution of drinking frequency per day

Valeurs calculées pour chaque chien au cours des trois programmes alimentaires et de la situation ad libitum.

La phase d'obscurité s'étend de 22 heures à 6 heures.

Calculated values for each dog during the three feeding protocols and in an ad libitum situation.

The dark phase was between 22.00 p.m. to 6.00 a.m.
- Chien $\mathrm{n}^{\circ} 1$.
Dog No. 1 .
Chien $n^{\circ} 2$.
Dog No. 2.
$\triangle \quad$ Chien $\mathrm{n}^{\circ} 3$.
Dog No. 3.

La flèche $(\downarrow)$ indique le moment du repas.

Arrow $(\downarrow)=$ meal-time. 


\section{3. - Répartition sur les 24 heures de la quantité moyenne d'eau ingérée par jour et par kilogramme de poids corporel}

En situation ad libitum (figure 4) on remarque que les tranches horaires de plus forte ingestion d'eau des chiens $\mathrm{n}^{\text {os }} 1$ et 2 se situent en phase d'obscurité alors que celles du $3^{*}$ chien se trouvent en phase d'éclairement. Comme précédemment, ces profils horaires sont profondément modifiés par l'imposition d'un repas unique par jour. Chez les trois chiens, l'ingestion d'eau, très forte dans la tranche horaire du repas ou dans la tranche suivante, diminue ensuite pour devenir très faible ou nulle en fin de phase d'éclairement et jusqu'au repas suivant.

La comparaison des profils obtenus au cours des trois programmes montre nettement que la diminution du poids du repas unique s'accompagne d'une réduction de cette évolution.

\section{4. - Poids, durée des prises d'eau}

Les résultats du tableau 2 montrent que les chiens $n^{\circ *} 1$ et 3 ont un comportement très proche. L'un et l'autre, lorsqu'ils passent de la situation ad libitum à l'alimentation programmée, diminuent significativement la taille de leurs prises d'eau ( $p<1$ p. 1000 ) ainsi que leur durée ( $\mathrm{p}$ compris entre 5 p. 100 et 1 p. 1000). Ces deux chiens ne diffèrent entre eux quau cours du programme alimentaire $\& B \gg$ (au seuil de 5 p. 100) pour le poids des prises d'eau.

Le chien $n^{\circ} 2$, quant à lui, se distingue nettement des deux autres. D'une part, il a tendance à augmenter le poids et la durée de ses prises d'eau lorsqu'il passe en alimentation programmée. D’autre part, quelle que soit la situation expérimentale étudiée, il effectue des prises d'eau toujours plus petites et de plus courte durée que les deux autres chiens ( $\mathrm{p}<1$ p. 1000 ).

\section{5. - Débits}

Dans les conditions ad libitum on observe toujours une corrélation entre le poids des prises d'eau et leur durée (tableau 2). Les débits d'ingestion moyens, exprimés en gramme d'eau par seconde, sont proches pour les trois chiens, puisqu'ils varient de 3,98 pour le chien $n^{\circ} 3$ à 4,50 pour le chien $n^{\circ} 1$.

Au cours des trois programmes les débits d'ingestion d'eau de chacun des chiens sont toujours inférieurs à ceux mesurés en situation ad libitum. (Les différences sont significatives pour les chiens $\mathrm{n}^{\text {os }} 1$ et 2.) De plus, la relation entre le poids des prises et leur durée devient plus étroite comme le fait apparaître le degré de signification des corrélations toujours supérieures à 1 p. 1000.

L'alimentation programmée modifie en sens inverse les débits d'ingestion des aliments. En effet, à l'exception du chien $n^{\circ} 1$ en programme « $A$ », les débits calculés sont toujours supérieurs à ceux de la situation ad libitum (tableau 3). 

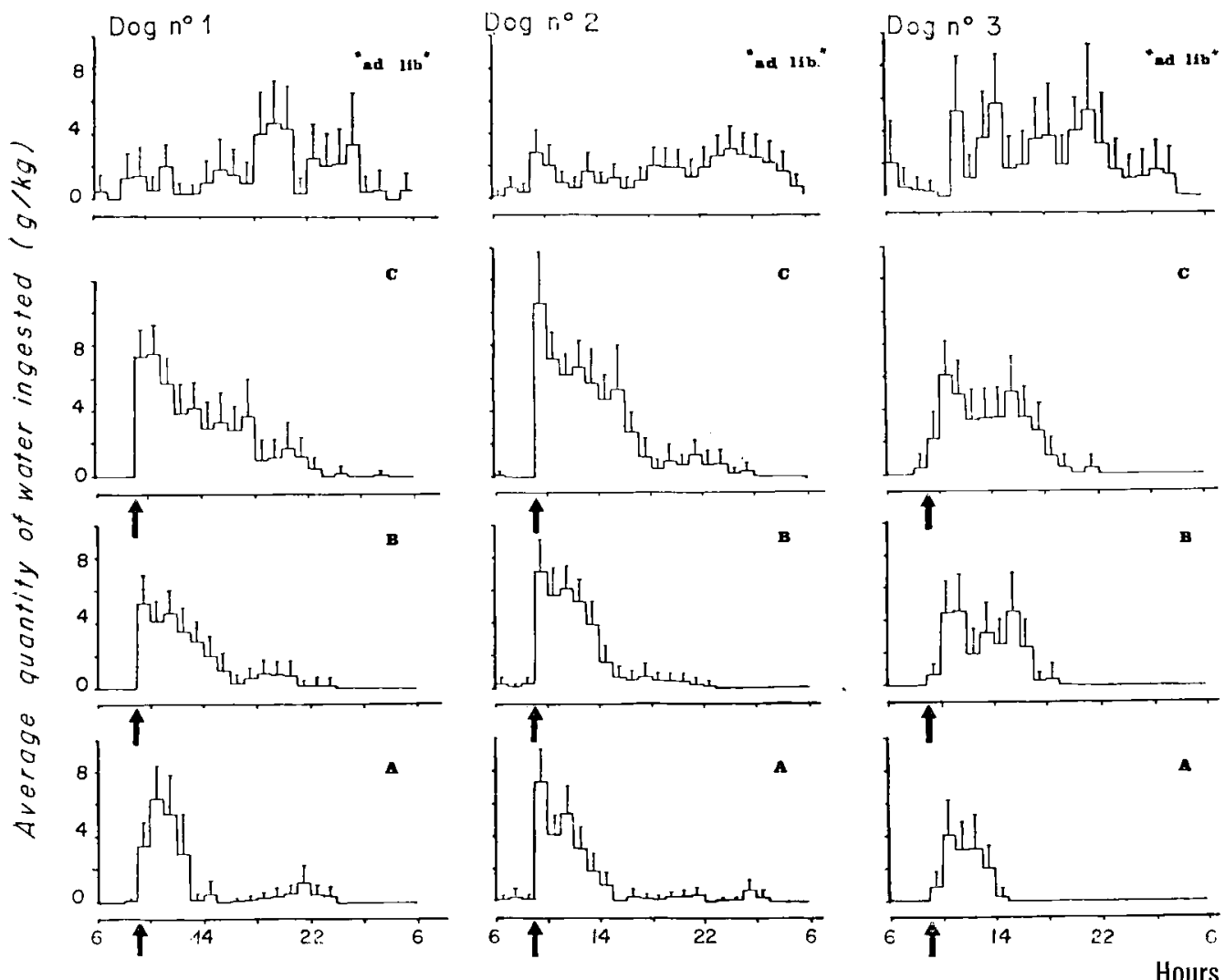

FIG. 4

Répartition sur les 24 heures de la quantité moyenne d'eau ingérée par jour et par kilogramme de poids corporel

Hourly distribution of the average quantity of water ingested daily

Valeurs calculées pour chaque chien au cours des trois programmes alimentaires (« $A$ », 《B», «C») et de la situation ad libitum.

Les barres verticales indiquent la limite supérieure de l'intervalle de confiance à 95 p. 100 . La phase d'obscurité s'étend de 22 heures à 6 heures.

Calculated values for each dog during the three feeding protocols ( $A \gg, 《 B »,\langle C 》)$ and in an ad libitum situation.

The vertical bars show the upper limit of the 95 p. 100 confidence interval.

The dark phase was between 22.00 p.m. to 6.00 a.m.

La flèche indique le moment du repas.

Arrow $=$ meal-time.

\section{6. - Relations poids-intervalles}

En situation $a b$ libitum il n'existe pas de liaison entre le poids des prises d'eau et l'intervalle libre qui les précède. Par contre des liaisons positives sont observées avec les intervalles qui leur font suite chez les chiens $n^{\text {o* }} 1$ et 2 (tableau 4).

En alimentation programmée on observe des liaisons négatives et une positive entre le poids des prises et les intervalles qui les précèdent chez les trois chiens et des liaisons toujours positives avec les intervalles après chez les chiens $n^{0 s} 1$ et 3 . 


\section{TABleau 2}

Valeurs moyennes des prises d'eau, des durées des prises d'eau et des débits d'ingestion en gramme par seconde

Mean water intake $(S)$, mean intake duration $(D)$ and $\frac{S}{D}$ (grammes per second)

\begin{tabular}{|c|c|c|c|c|}
\hline & & $\begin{array}{c}\operatorname{Dog} n^{\circ} \\
\text { Chien } n^{\circ} \\
1\end{array}$ & $\begin{array}{c}\operatorname{Dog} n^{\circ} \\
\text { Chien } \mathrm{n}^{\circ} \\
2\end{array}$ & $\begin{array}{c}\operatorname{Dog} n^{\circ} \\
\text { Chien } n^{\circ} \\
3\end{array}$ \\
\hline \multirow{4}{*}{$\begin{array}{c}\text { Poids } \\
\text { d'une prise d'eau } \\
\text { Weight } \\
\text { of one water intake } \\
\text { (G) } \\
\mathrm{g} \pm \mathrm{SEM}\end{array}$} & A & $162,73 \pm 8,65$ & $88,57 \pm 2,68$ & $151,46 \pm 9,97$ \\
\hline & B & $148,92 \pm 6,12$ & $97,57 \pm 2,72$ & $175,88 \pm 7,13$ \\
\hline & $\mathrm{C}$ & $172,34 \pm 4,92$ & $106,49 \pm 2,57$ & $162,01 \pm 4,23$ \\
\hline & $\begin{array}{c}A d \\
\text { libitum }\end{array}$ & $244,21 \pm 12,43$ & $95,96 \pm 2,97$ & $224,83 \pm 8,44$ \\
\hline \multirow{4}{*}{$\begin{array}{c}\text { Durée } \\
\text { d'une prise d'eau } \\
\text { Water intake length } \\
\text { (D) } \\
\text { s } \pm \text { SEM }\end{array}$} & $\mathrm{A}$ & $45,88 \pm 1,63$ & $25,10 \pm 0,77$ & $42,20 \pm 2,10$ \\
\hline & $\mathrm{B}$ & $42,37 \pm 1,15$ & $27,76 \pm 0,76$ & $45,71 \pm 1,59$ \\
\hline & $\mathrm{C}$ & $47,71 \pm 1,01$ & $31,40 \pm 0,76$ & $45,21 \pm 1,01$ \\
\hline & $\underset{\text { libitum }}{A d}$ & $61,77 \pm 7,46$ & $23,90 \pm 0,73$ & $63,36 \pm 6,28$ \\
\hline \multirow{4}{*}{$\begin{array}{c}\text { Débit } \\
\text { d'une prise d'eau } \\
\text { Water intake rate } \\
\text { G/D }\end{array}$} & A & $3,59 \pm 0,219$ & $3,56 \pm 0,059$ & $3,59 \pm 0,131$ \\
\hline & B & $3,40 \pm 0,094$ & $3,52 \pm 0,051$ & $3,85 \pm 0,108$ \\
\hline & $\mathrm{C}$ & $3,62 \pm 0,078$ & $3,44 \pm 0,041$ & $3,58 \pm 0,051$ \\
\hline & $\begin{array}{c}\text { Ad } \\
\text { libitum }\end{array}$ & $4,51 \pm 0,161$ & $4,03 \pm 0,057$ & $3,98 \pm 0,129$ \\
\hline \multirow{4}{*}{$\begin{array}{l}\text { Coefficient } \\
\text { de corrélation } \\
\text { Correlation } \\
\text { coefficient } \\
(\mathrm{G}-\mathrm{D})\end{array}$} & A & $0,754^{* * *}(91)$ & $0,919 * * *(165)$ & $0,753 * * *(41)$ \\
\hline & B & $0,708^{* * * *}(168)$ & $0,924 * * *(211)$ & $0,764 * * *(70)$ \\
\hline & $\mathrm{C}$ & $0,652^{* * *}(200)$ & $0,930^{* * * *}(217)$ & $0,840^{* * *}(103)$ \\
\hline & $\begin{array}{c}A d \\
\text { libitum }\end{array}$ & $0,257^{*} \quad(95)$ & $0,943 * * * *(226)$ & $0,289 * *$ \\
\hline
\end{tabular}

$* \mathrm{p}<0,05$.

*** $\mathrm{p}<0,01$.

$* * * \quad \mathrm{p}<0,001$.

Valeurs calculées pour chaque chien au cours des trois programmes alimentaires et de la situation ad libitum. En bas, les valeurs des coefficients de corrélation : poids d'eau ingéré - durée de la prise.

Les chiffres entre parenthèses indiquent le nombre de paires corrélées.

Calculated values for each dog during the three feeding protocols and in an ad libitum situation.

The lower third of the table shows the correlation coefficient between weight and duration of water intake.

The numbers in parentheses indicate the number of paired values. 


\section{Tableau 3}

Valeurs moyennes des débits d'ingestion des aliments (en grammes par seconde) Average food intake rate ( $g$ per second)

\begin{tabular}{c|c|c|c}
\hline & $\begin{array}{c}\text { Dog } n^{\circ} \\
\text { Chien } n^{\circ} \\
1\end{array}$ & $\begin{array}{c}\text { Dog } n^{\circ} \\
\text { Chien } n^{\circ} \\
2\end{array}$ & $\begin{array}{c}\text { Dog } n^{\circ} \\
\text { Chien } \mathbf{n}^{\circ} \\
3\end{array}$ \\
\hline $\mathrm{A}$ & $\begin{array}{c}0.61 \pm 0,018 \\
(40)\end{array}$ & $\begin{array}{c}1,21 \pm 0,021^{* * *} \\
(36)\end{array}$ & $\begin{array}{c}0,72 \pm 0,024^{*} \\
(47)\end{array}$ \\
\hline $\mathrm{B}$ & $\begin{array}{c}0,68 \pm 0,012^{* * *} \\
(48)\end{array}$ & $\begin{array}{c}1,32 \pm 0,035^{* * *} \\
(35)\end{array}$ & $\begin{array}{c}0,95 \pm 0,028^{* * *} \\
(52)\end{array}$ \\
\hline $\mathrm{C}$ & $\begin{array}{c}0,76 \pm 0,016^{* * *} \\
(36)\end{array}$ & $\begin{array}{c}1,38 \pm 0,016^{* * *} \\
(34)\end{array}$ & $\begin{array}{c}0,98 \pm 0,027^{* * *} \\
(43)\end{array}$ \\
\hline Ad libitum & $\begin{array}{c}0,59 \pm 0,021 \\
(71)\end{array}$ & $\begin{array}{c}0,66 \pm 0,014 \\
(116)\end{array}$ & $\begin{array}{c}0,65 \pm 0,016 \\
(105)\end{array}$ \\
\hline
\end{tabular}

* $\mathrm{p}<0,05$.

**** $\mathrm{p}<0,001$.

Valeurs calculées pour chaque chien au cours des trois programmes alimentaires et de la situation ad libitum.

Les * indiquent les résultats des comparaisons du débit moyen d'ingestion d'eau en situation ad libitum avec celui de chaque programme alimentaire pour chaque chien.

Les chiffres entre parenthèses indiquent le nombre de données. situation.

Calculated values for each dog during the three feeding protocols and in an ad libitum

The * indicate comparison of food intake rate $(\mathrm{g} / \mathrm{s})$ in the ad libitum situation and in protocols $《 A », \ll B »$ and $《 C »$, for each dog.

The numbers in parentheses indicate the number of values.

\section{Discussion}

\section{Résultats journaliers}

De nombreux travaux ont été consacrés aux effets de la restriction alimentaire sur le comportement de prise d'eau. Dans ces conditions, l'existence d'une relation linéaire entre les consommations journalières d'eau et d'aliments a été mise en évidence chez le Rat (Strominger, 1947 ; Cizek \& Nocenti, 1965 ; Kutscher, 1969) et chez le Chien (Towbin, 1955; Cizek, 1959). Dans le présent travail seul le chien $\mathrm{n}^{*} 3$ présente une évolution analogue à celle décrite par TowBIN (1955) et CizeK (1959). En effet, pour les chiens $n^{\circ s} 1$ et 2 le rapport quantité d'eau sur quantité d'aliments ingérés quotidiennement est toujours supérieur, au cours des trois programmes alimentaires, à celui obtenu en situation ad libitum. La réduction hydrique spontanée n'est donc pas proportionnelle à la réduction alimentaire imposée. L'examen des comportements de prise d'eau et d'aliments en situation ad libitum des trois chiens ne permet pas d'expliquer ces différences en situation de contrainte. La surconsommation relative d'eau observée chez les chiens $n^{o s} 1$ et 2 pourrait être comparée à la polydipsie décrite par FALK $(1961,1969)$ et Roehrs, Allen \& Porter (1976) 
Tableau 4

Valeurs moyennes des prises d'eau et des intervalles entre les prises d'eau

Mean water intake and pre- and post-drinking intervals

\begin{tabular}{|c|c|c|c|c|}
\hline & & $\begin{array}{c}\operatorname{Dog} n^{\circ} \\
\text { Chien } n^{\circ} \\
1\end{array}$ & $\begin{array}{c}\operatorname{Dog} n^{\circ} \\
\text { Chien } n^{\circ} \\
2\end{array}$ & $\begin{array}{c}\operatorname{Dog} n^{\circ} \\
\text { Chien } n^{\circ} \\
3\end{array}$ \\
\hline \multirow{4}{*}{$\begin{array}{c}\text { Poids } \\
\text { d'une prise d'eau } \\
(\mathrm{P}) \\
\text { Weight } \\
\text { of the water intake } \\
\mathrm{g} \pm \mathrm{SEM}\end{array}$} & $\mathrm{A}$ & $161,67 \pm 9,13$ & $88,66 \pm 2,72$ & $135,23 \pm$ \\
\hline & B & $146,26 \pm 6,46$ & $97,57 \pm 2,74$ & $171,96 \pm 8,25$ \\
\hline & $\mathrm{C}$ & $172,91 \pm 4,95$ & $107,17 \pm 2,59$ & $159,91 \pm$ \\
\hline & $\underset{\text { libitum }}{A d}$ & $241,99 \pm 12,83$ & $95,97 \pm 3,02$ & $221,82 \pm$ \\
\hline \multirow{4}{*}{$\begin{array}{c}\text { Durée de l'intervalle } \\
\text { entre deux prises } \\
\text { d'eau } \\
\text { Length } \\
\text { of the interval } \\
\text { between } \\
\text { two water intakes } \\
\text { min } \pm \text { SEM }\end{array}$} & A & $352,45 \pm 44,31$ & $191,68 \pm 22,98$ & $801,35 \pm 126,34$ \\
\hline & $\mathbf{B}$ & $255,73 \pm 28,51$ & $166,19 \pm 18,61$ & $489,69 \pm 63,35$ \\
\hline & $\mathrm{C}$ & $183,25 \pm 18,45$ & $106,57 \pm 13,17$ & $288,35 \pm 37,39$ \\
\hline & 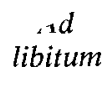 & $337,70 \pm 32,61$ & $161,18 \pm 10,60$ & $363,51 \pm 28,55$ \\
\hline \multirow{4}{*}{$\begin{array}{c}\text { Coefficient } \\
\text { de corrélation } \mathrm{P}- \\
\text { Intervalle après } \\
\text { Correlation } \\
\text { coefficient } P- \\
\text { post-interval }\end{array}$} & A & $0,278^{*}$ & $-0,081$ & $0,447^{*}(26)$ \\
\hline & B & $0,290^{* * *}(154)$ & $-0,049 \quad(209)$ & $0,339 *(55)$ \\
\hline & $\mathrm{C}$ & $0,156^{*} \quad(190)$ & $-0,011$ & $0,050 \quad(89)$ \\
\hline & $\begin{array}{c}A d \\
\text { libitum }\end{array}$ & $0,479 * * *(90)$ & $0,265^{* * *}(222)$ & $0,032 \quad(80)$ \\
\hline \multirow{4}{*}{$\begin{array}{c}\text { Coefficient } \\
\text { de corrélation } \mathrm{P}- \\
\text { Intervalle avant } \\
\text { Correlation } \\
\text { coefficient } P- \\
\text { pre-interval }\end{array}$} & A & $-0,311^{* *}(84)$ & $-0,292^{* * *}(161)$ & $0,402 *(26)$ \\
\hline & B & $-0,074 \quad(152)$ & $-0,288^{* * *}(207)$ & $0,090 \quad(55)$ \\
\hline & $\mathrm{C}$ & $-0,038 \quad(191)$ & $-0,330^{* *} \quad(210)$ & $-0,210^{*}(93)$ \\
\hline & $\underset{\text { libitum }}{A d}$ & $-0,160$ & $\longrightarrow 0,118$ & $-0,067 \quad(87)$ \\
\hline
\end{tabular}

$* \quad \mathrm{p}<0,05$

** $\quad \mathrm{p}<0,01$.

*** $\mathrm{p}<0,001$.

Valeurs observées pour chaque chien au cours des trois programmes alimentaires et de la situation ad libitum.

En bas les valeurs des coefficients de corrélation : poids d'eau ingérée - durée de l'intervalle avant la prise, poids d'eau ingérée - durée de l'intervalle après la prise.

Les chiffres entre parenthèses indiquent le nombre de paires corrélées.

Calculated values for each dog during the three feeding protocols and in an ad libitum situation.

The lower third of the table shows the correlation coefficient between weight of water intake and pre- and post-drinking intervals.

The numbers in parentheses indicate the number of paired values. 


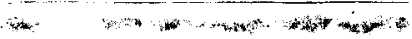

chez le Rat soumis à un conditionnement opérant à renforcement alimentaire. Cependant de nombreuses différences opposent les situations expérimentales. En effet, si dans les deux cas la motivation à ingérer les aliments est forte de par la privation préalable, dans l'expérience de FALK le débit d'ingestion des aliments est instrumentalement très ralenti alors qu'il est toujours spontanément augmenté par rapport à la situation ad libitum chez nos chiens. Par ailleurs, la polydipsie induite des rats de FALK les conduit à ingérer un volume d'eau supérieur au triple de celui qu'ils ingèrent lorsqu'ils peuvent régler spontanément leur débit d'ingestion des aliments; alors que les chiens $\mathrm{n}^{\text {os }} 1$ et 2 boivent respectivement 128 et 148 p. 100 du volume ingéré en situation ad libitum.

\section{Cette surconsommation pourrait être rattachée à deux origines :}

1) pour les programmes les plus restrictifs, la réduction de la prise d'eau ne porte pas sur la totalité du volume ingéré en situation ad libitum car une partie de la prise d'eau quotidienne est irréductible, indépendante de la prise solide, comme cela a été montré dans les expériences de jeûne alimentaire chez le Rat (CizEK \& Nocenti, 1965; Fitzsimons \& Le Magnen, 1969; Oatley, 1971) et chez le Chien (CIZEK, 1959 ; Ardisson et al., 1975);

2) pour le programme $C$, les résultats obtenus par Deaux, Sato \& Kakolewski en 1970 suggèrent qu'elle pourrait être reliée à l'augmentation forte et précoce de la pression osmotique du plasma dans les minutes qui suivent l'ingestion du repas unique. En effet, dans cette situation, l'ingestion rapide d'un volume important d'aliments entraîne une stimulation intense des récepteurs buccaux et cesophagiens, une forte distension gastrique et un déplacement important de l'eau du milieu intérieur vers la lumière intestinale pour permettre la dilution puis l'absorption des molécules nutritionnelles (Gregersen, 1932; LepKovsky et al., 1957). Pour Lepkovsky et al. (1957) l'ingestion d'eau observée chez le Rat à proximité immédiate du repas a pour rôle de prévenir ou d'atténuer cette augmentation de la pression osmotique du milieu intérieur. Cependant, cet auteur n'observe pas chez cette espèce de véritable polydipsie. Par contre, ce phénomène a été constaté par KuTscher (1969) chez la Gerbille et le Hamster, par Crow (1964) chez le Rat et par CizeK (1954, 1961) chez le Cobaye et chez le Lapin mâle. On retrouve donc chez le Chien comme chez de nombreuses espèces une surconsommation d'eau lorsque la prise d'aliment est réalisée sous la forme de repas d'un volume inhabituellement grand.

\section{Profils horaires}

Lorsque les chiens ne font qu'un repas par jour, en phase d'éclairement, les patterns de prise d'eau sont complètement remodelés par rapport à la situation $a d$ libitum. Les prises d'eau sont déclenchées par le repas, regroupées dans les heures qui le suivent et les prises nocturnes disparaissent. Déjà Gregersen en 1932 avait noté que des chiens vivant dans des cages à métabolisme et recevant un repas par jour concentraient leurs prises d'eau dans les 5 à 6 heures après le repas. De même, plus récemment Golob, O'Connor \& PotTs (1977) ont montré que des chiens recevant un repas par jour à $17 \mathrm{~h}$ boivent dans les huit heures qui suivent.

Bien qu'obtenus dans des conditions expérimentales différentes les résultats de cet auteur sont très proches des nôtres. En effet, si le poids du repas est doublé, ses 
chiens boivent davantage et plus longtemps après la prise alimentaire comme le font nos chiens lorsque la ration alimentaire augmente. Ainsi, chez le Chien, le comportement de prise d'eau semble, dans des circonstances de contrainte alimentaire, déterminé étroitement par les modalités de prise d'aliments. En situation inverse, Prud'hon et al. (1975) chez le Lapin et Bolles (1961) chez le Rat ont montré que le comportement alimentaire spontané est moins fondamentalement modifié par une programmation hydrique. Une étude analogue chez le Chien devrait permettre une comparaison plus précise des interrelations entre les comportements de prise d'eau et d'aliments chez ces diverses espèces.

\section{Relations}

Quel que soit le programme alimentaire considéré, il existe une diminution du débit d'ingestion d'eau de chacun des trois chiens par rapport à la situation ad libitum. L'imposition d'un seul repas par jour semble donc influencer ce paramètre. Cette diminution pourrait s'expliquer par un effet inhibiteur lié à la forte distension gastrique déterminée par l'ingestion rapide du repas unique. En effet, les débits d'ingestion des granulés sont toujours significativement supérieurs à ceux mesurés en situation ad libitum.

Les liaisons entre le poids et la durée des prises d'eau ainsi que celles entre le poids des prises d'eau et les intervalles de temps qui leur font suite persistent en alimentation programmée. On retrouve donc, chez deux chiens sur trois, et pour les prises d'eau, les relations fondamentales précédemment décrites, pour les prises d'aliments, chez le Rat (Le Magnen \& Tallon, 1966), chez le Lapin (Sanderson \& VanDERWEele, 1975) et le Singe (NATELson \& Bonbright, 1978).

Ces relations ne sont pas systématiquement présentes chez nos trois chiens, ce qui traduit, à nouveau, l'importance des différences interindividuelles et l'intérêt d'une étude séparée de chaque sujet, même lorsque l'on utilise des souches très homogènes comme l'a montré BERnSTEin (1975). En effet, cet auteur a mis en évidence que, chez le Rat blanc, les relations décrites par LE MAGNEN \& TaLlon (1966), à partir de données provenant d'une population, n'étaient retrouvées, systématiquement, que chez trois individus sur six. Quant aux corrélations négatives entre le poids des prises d'eau et les intervalles qui les précèdent, elles traduisent, en alimentation programmée, l'évolution de la fréquence de ces prises après le repas unique.

En conclusion, en situation ad libitum les comportements de prise d'eau des trois chiens de cette étude présentent de fortes différences interindividuelles portant sur la taille, la fréquence et la répartition des prises.

En situation de restriction alimentaire on constate une convergence des comportements concernant surtout la répartition horaire des prises et leurs débits, mais peu de modifications de la dimension des prises propre à chacun des trois chiens. De même, les relations fondamentales entre le poids et la durée des prises d'eau ainsi que celles entre le poids des prises d'eau et les intervalles de temps qui leur font suite persistent en alimentation programmée.

Ces résultats indiquent que, lorsque les conditions du milieu deviennent contraignantes, les importantes différences interindividuelles que l'on observe spontanément 
en situation ad libitum s'estompent partiellement. Des situations plus contraignantes (jeûnes) devraient permettre de vérifier si ces nouveaux comportements, apparemment caractéristiques de l'espèce, persistent voire s'accentuent lorsque les degrés de liberté de l'organisme diminuent.

Accepté pour publication en février 1981.

Cette étude a bénéficié de l'aide du C.N.R.S. (A.T.P. 2807).

\section{Summary}

\section{Effects of programmed feeding on drinking behavior in dogs}

Drinking behavior in three dogs was studied according to two experimental situations : 1) ad libitum feeding, 2) only one meal per day representing a fraction of the dog's daily voluntary feed intake, i.e. 30 p. 100 in protocol A, 60 p. 100 in protocol B and 90 p. 100 in protocol $\mathrm{C}$.

Comparison of the results of these two situations shows that intake of one only meal per day leads to :

1) a persistence of the individual variations in mean water intake;

2) a significant correlation between the weight of water consumed and the post-intake interval in two dogs (table 4);

3) a monomorphic change in the spontaneous drinking patterns : they are grouped in the hours following the meals (fig. 3);

4) a) an increase in the food intake rate and a decrease in the water intake rate in the three dogs (tables 2 and 3); (table 1).

b) an increase in the quantity of water consumed per gramme of feed in two dogs

\section{Références bibliographiques}

Adolph E.F., 1947. Urges to eat and drink in rats. Am. J. Physiol., 151, 110-125.

Ardisson J.L., Dolisi C., Camous J.P., Ozon C., 1975. Incidences des modalités d'alimentation sur la prise d'eau chez le Chien. J. Physiol., Paris, 70, 139-148.

Ardisson J.L., Ozon C., Dolisi C., Crenesse D., 1979. Enregistrement en continu du comportement alimentaire et moteur du Chien en semi-liberté. J. Physiol., Paris, 75, 89 A.

Bernstein I.L., 1975. Relationship between activity, rest, and free feeding in rats. J. Comp. Physiol. Psychol., 89, 253-257.

Bing F.C., Mendel L.B., 1931. The relationship between food and water intakes in mice. Am. J. Physiol., 98, 169-179.

Bolles R.C., 1961. The interaction of hunger and thirst in the rat. J. Comp. Physiol. Psychol., 54, 580-584.

Cizek L.J., 1954. Total water content of laboratory animals with special reference to volume of fluid within the lumen of the gastrointestinal tract. Am. J. Physiol., 179, 104-110.

Cizek L.J., 1959. Long-term observations on relationship between food and water ingestion in the dog. Am. J. Physiol., 197, 342-346. 
Cizek L.J., 1961. Relationship between food and water ingestion in the rabbit. Am. J. Physiol., 201, 557-566.

Cizek L.J., Nocenti M.R., 1965. Relationship between water and food ingestion in the rat. Am. J. Physiol., 208, 615-620.

Crow L.T., 1964. Sub-commissural organ, lateral hypothalamus and dorsal longitudinal fasciculus in water and salt metabolism. Intern. Sympos. on Thirst in the Regulation of body water. 1st. Thirst. Pergamon, Oxford, England; M.J. Wayner ed., 473-481.

Deaux E., Sato E., Kakolewski J.W., 1970. Emergence of systemic cues evoking food associated drinking. Physiol. Behav., 5, 1177-1179.

FaLK J.L., 1961. Production of polydipsia in normal rats by an intermittent food schedule. Science, 133, 195-196.

FALK J.L., 1969. Conditions producing psychogenic polydipsia in animals. Ann. N.Y. Acad. Sci., 157, 569-593.

Fitzsimons J.T., Le Magnen J., 1969. Eating as a regulatory control of drinking in the rat. J. Comp. Physiol. Psychol., 67, 273-283.

Golob P., O'Connor W.J., PotTs D.J., 1977. Post-prandial drinking by dogs. Quart. Jl. Exp. Physiol., 62, 275-285.

Gregersen M.I., 1932. Studies on the regulation of water intake. II - Conditions affecting the daily water intake of dogs as registered continuously by a potometer. Am. J. Physiol., 103, 344-349.

Kutscher C.L., 1969. Species differences in the interaction of feeding and drinking. Ann. N.Y. Acad. Sci., 157, 539-552.

Le Magnen J., Tallon S., 1966. La périodicité spontanée de la prise d'aliments ad libitum du Rat blanc. J. Physiol., Paris, 58, 323-349.

Lepkovsky S., Lyman R., Gleming D., Nagumo M., Dimick M., 1957. Gastrointestinal regulation of water and its effect on food intake and rate of digestion. Am. J. Physiol., 188, 327-331.

Levitsky D.A., 1974. Feeding conditions and intermeal relationships. Physiol. Behav., 12, 779-787.

Natelson B.H., Bonbright J.C., 1978. Patterns of eating and drinking in monkeys when food and water are free and when they are earned. Physiol. Behav., 21, 201-213.

OAtLey K., 1971. Dissociation of the circadian drinking pattern from eating. Nature, 229, 494-496.

Prud'hon M., Cherubin M., Carles Y., Goussopoulos J., 1975. Effets de différents niveaux de restriction hydrique sur l'ingestion d'aliments solides par le Lapin. Ann. Zootech., 24, 299-310.

Roehrs T., Allen J.D., Porter J.H., 1976. Acquisition of schedule-induced polydipsia in rats with no prior drinking experience. Physiol. Behav., 17, 1011-1013.

SANDerson J.D., Vanderweele D.A., 1975. Analysis of feeding patterns in normal and vagotomized rabbits. Physiol. Behav., 15, 357-364.,

Strominger J.L., 1947. The relation between water intake and food intake in normal rats and in rats with hypothalamic hyperphagia. Yale. J. Biol. Med., 19, 279-288.

TowBin E.J., 1955. Thirst and hunger behavior in normal dogs and the effects of vagatomy and sympathectomy. Am. J. Physiol., 182, 377-382.

Verplanck W.S., Hayes J.R., 1953. Eating and drinking as a function of maintenance schedule. J. Comp. Physiol. Psychol., 46, 327-333. 